\title{
Poultry Ownership and Genetic Antibiotic Resistance Determinants in the Gut of Preschool Children
}

\author{
Jessica M. Brogdon, ${ }^{1 \star}$ Ali Sié,${ }^{2}$ Clarisse Dah, ${ }^{2}$ Lucienne Ouermi,${ }^{2}$ Boubacar Coulibaly, ${ }^{2}$ Elodie Lebas, ${ }^{1}$ Lina Zhong, ${ }^{1}$ Cindi Chen, ${ }^{1}$ \\ Thomas M. Lietman, ${ }^{1,3,4}$ Jeremy D. Keenan, 1,3,4 Thuy Doan, ${ }^{1,3}$ and Catherine E. Oldenburg ${ }^{1,3,4}$ \\ ${ }^{1}$ Francis I Proctor Foundation, University of California, San Francisco, San Francisco, California; ${ }^{2}$ Centre de Recherche en Santé de Nouna, Nouna, \\ Burkina Faso; ${ }^{3}$ Department of Ophthalmology, University of California, San Francisco, San Francisco, California; ${ }^{4}$ Department of Epidemiology and \\ Biostatistics, University of California, San Francisco, San Francisco, California
}

\begin{abstract}
Zoonotic transmission is likely a pathway for antibiotic resistance. Data from a randomized trial of pediatric antibiotic administration were secondarily evaluated to determine if poultry ownership was significantly associated with the presence of gut genetic antibiotic resistance determinants among 118 children in Burkina Faso. Antimicrobial resistance (AMR) determinants were classified using DNA sequencing. We measured the relationship between genetic resistance determinants and chicken ownership using a logistic regression model adjusted for confounding variables. Children in households reporting poultry ownership had four times the odds of tetracycline resistance determinants in the gut compared with those without household poultry (odds ratio [OR]: 4.08, 95\% Cl: $1.08-15.44, P=0.04$ ). There was no statistically significant difference found for other antibiotic classes. Understanding the origins of antibiotic resistance may help spur the development of interventions to combat the global AMR crisis.
\end{abstract}

A growing body of evidence suggests animal-human transmission of antibiotic resistance is a major spillover event occurring worldwide. ${ }^{1-4}$ Antimicrobial resistance (AMR) is one of the top threats to global health today, particularly in lowand middle-income countries (LMICs). ${ }^{5,6}$ Antimicrobial resistance renders antibiotics ineffective against dangerous bacterial infections and can lead to an increased risk of mortality. ${ }^{7}$ Often antibiotics are suboptimally used in LMICs for livestock prophylaxis and growth stimulation. Empirical antibiotic use for domesticated animals may pose risks for AMR in the developing world, where livestock lives more closely with humans. ${ }^{8}$ The misuse of antimicrobials contributes to the emergence of resistance by creating selection pressure for resistant genes. ${ }^{4,8}$ Understanding the origins of antibiotic resistance may help spur the development of interventions to combat AMR.

In Burkina Faso, poultry are an important source of food and livelihood for many households. Poultry live within close proximity of human living quarters and commonly roam freely within household compounds. ${ }^{9}$ Chicken-owning households have frequent interactions with poultry while caring for them and likely encounter exposure to chicken feces, which is a risk factor for enteric disease. ${ }^{10}$ Chicken ownership may pose additional human health risks. Here, we investigate if poultry ownership is significantly correlated with genetic antibiotic resistance determinants in the gut among children aged 659 months in rural Burkina Faso.

Data from a randomized pediatric antibiotic trial were secondarily evaluated for this analysis. Detailed methods for the parent trial have been previously reported. ${ }^{11-14}$ The study occurred in northwestern Burkina Faso in July 2017. Communities were selected that had at minimum 120 households with at least 2 children between the ages of 6-59 months. Household children received one of three antibiotics or

*Address correspondence to Jessica M. Brogdon, Francis I Proctor Foundation, University of California, San Francisco, 490 Illinois St. Floor 2, San Francisco, CA 94158. E-mail: jessica.brogdon@ucsf.edu placebo. One hundred eighteen children were included for the purposes of this sub-analysis.

A caregiver survey was administered at baseline with questions related to child health and household qualities. Household socioeconomic status was determined by household resources and if the child recently visited a health facility. Household food insecurity was defined by asking if the child went to bed hungry and if the household had enough food. Questions about the child's diet and breastfeeding status were included to define the child's dietary diversity. Caregivers also reported the number of poultry owned by the household.

Anthropometric measurements were collected from children at baseline, including height, weight, and mid-upper arm circumference. Methods for obtaining anthropometric measurements are previously reported for the parent trial. ${ }^{12,13}$ Weight-for-height $Z$, weight-for-age $Z$, and height-for-age $Z$ scores were calculated based on the 2006 WHO standards.

Rectal swab samples were collected from all household children at baseline and 5 days following antibiotic treatment. Examiners inserted a cotton swab into the child's anus $1-3 \mathrm{~cm}$ and rotated $360^{\circ}$. Swabs were placed into a stool nucleic acid collection transport tube containing Norgen Stool Preservative (Norgen, Ontario, Canada). RNA and DNA were preserved in the sample by the Norgen media, and growth of other organisms was prohibited. To evaluate the resistome, or the collection of genetic resistance determinants in the gut, we used metagenomic deep sequencing methods as previously described, which identified all genetic resistance determinants in the sample. ${ }^{14}$ These determinants were then classified at the class and gene level for analysis.

The primary exposure of interest was chicken ownership, which was dichotomized as yes or no if the household owned poultry. Descriptive characteristics were summarized, with medians and interquartile ranges (IQR) for continuous variables and proportions for categorical variables. A series of logistic regression models were used to measure the relationship between genetic resistance determinants and chicken ownership. We constructed 12 models which included one bivariate (unadjusted) and one multivariable 
TABLE 1

Characteristics of children included

Chickens in household $(N=106)$ No chickens in household $(N=12)$

\begin{tabular}{lcc}
\hline Median child's age (IQR) (months) & $35.5(22-51)$ & $36(20-50)$ \\
Female gender, $N(\%)$ & $51(48.1)$ & $5(41.7)$ \\
Median dietary diversity score (IQR) & $7(5-8)$ & $5(3.5-6)$ \\
Breastfeeding, $N(\%)$ & $27(25.5)$ & $3(25.0)$ \\
Household lacks resources, $N(\%)$ & $20(18.9)$ & $5(41.7)$ \\
Median number of chickens owned (IQR) & $6.5(3-15)$ & $0(0)$ \\
Median weight-for-height Z-score (IQR) & $-0.4(-1.0$ to 0.3$)$ & $-0.4(-0.9$ to 0.3$)$ \\
Median height-for-age Z-score (IQR) & $-0.9(-1.6$ to -0.3$)$ & $-1.0(-2.0$ to -0.7$)$ \\
Median weight-for-age Z-score (IQR) & $-0.8(-1.5$ to -0.3$)$ & $-0.8(-1.6$ to -0.3$)$ \\
\hline
\end{tabular}

(adjusted) per antibiotic class. Six antibiotic classes in total were evaluated. The multivariable models were adjusted for potentially confounding factors including age, gender, recent healthcare utilization, and household food insecurity. The standard error was adjusted to account for the fact that children within the same household are more similar to each other than they are to children outside of the household. As a sensitivity analysis, we analyzed the number of chickens owned by the household as a continuous variable. Analyses were performed in Stata version 15.1 (StataCorp, College Station, TX).

Baseline characteristics were similar among the 118 children living in households with or without poultry (Table 1). The median age (interquartile range) was 36 (21-51) months, and $48 \%$ of the children were female. Among this population, $90 \%$ lived in a household reporting to own at least one chicken. A greater percentage of children living in households without poultry reported a lack of household resources (41.7\%).

Antibiotic resistance was common among children living in households with and without poultry (Table 2). In bivariate logistic regression models, there were no significant differences between poultry ownership and the presence of antibiotic resistance determinants for several antibiotic classes including tetracycline, macrolides, and sulfonamides. After adjusting for the confounding factors (age, gender, recent healthcare utilization, and household food insecurity), the multivariable model demonstrated a statistically significant relationship between chicken ownership and the presence of tetracycline resistance. Children living in households reporting poultry ownership had four times the odds of tetracycline resistance determinants in the gut compared with those without household poultry (OR: $4.08,95 \% \mathrm{Cl}: 1.08-15.44, P=0.04)$. Resistance to other antibiotic classes was not statistically significant in the multivariable model, but resistance was increased in households with poultry in classes such as beta-lactams (OR: 2.75, 95\% Cl: 0.75-10.11, $P=0.13$ ), fluoroquinolones (OR: 1.87, 95\% Cl: $0.40-8.82, P=0.43$ ), and sulfonamides (OR: $1.53,95 \% \mathrm{Cl}: 0.32-7.29, P=0.60$ ). Analyzing the number of chickens owned by the household as a continuous variable did not qualitatively change the results.

These findings suggest children living in households with poultry have higher odds of gut genetic AMR determinants to tetracycline than those in households without poultry. These results are consistent with other studies that demonstrate the spread of AMR between poultry and humans. ${ }^{4,15,16,18}$ A Ugandan trial found small-scale rural chicken farmers acquired more AMR genes than unexposed participants. ${ }^{15}$ Presumably, chicken-owning households have more frequent interaction and exposure to chickens than non-chickenowning households. Children residing in households with poultry may be exposed to chicken feces containing high levels of antibiotic residue because tetracycline is often fed to chickens in this setting prophylactically. ${ }^{17,19}$ Antibiotic resistance determinants found within children are especially concerning because AMR is associated with difficult-to-treat infections and increased mortality. ${ }^{20}$ Overall, our findings suggest that living in close proximity to poultry may have detrimental effects on child health.

Several limitations should be taken into consideration for this analysis. The small sample size for this investigation created low statistical power and could inflate the effect size. Both poultry ownership and the presence of resistance determinants were common. As a result, the $95 \%$ Cls were wide. Self-reporting bias could have been introduced because chicken ownership was self-reported by the caregiver and was not verified by the research team. This analysis may not be representative for populations outside of this setting where poultry do not live in such close quarters with humans, and may therefore present low external validity. Analysis of poultry

TABLE 2

Association between poultry ownership and gut genetic antibiotic resistance determinants among children in Burkina Faso

\begin{tabular}{|c|c|c|c|c|c|c|}
\hline & \multicolumn{2}{|c|}{ Resistant, $N(\%)$} & \multicolumn{2}{|c|}{ Bivariate } & \multicolumn{2}{|c|}{ Multivariable } \\
\hline & Chickens & No chickens & OR $(95 \% \mathrm{Cl})$ & $P$-value & aOR $(95 \% \mathrm{Cl})$ & $P$-value \\
\hline Tetracycline & $93(87.7)$ & $9(75.0)$ & 2.38 (0.62-9.05) & 0.20 & $4.08(1.08-15.44)$ & 0.04 \\
\hline Beta-lactam & 89 (84.0) & $9(75.0)$ & $1.75(0.47-6.45)$ & 0.40 & $2.75(0.75-10.11)$ & 0.13 \\
\hline Sulfonamides & $27(25.5)$ & $2(16.7)$ & $1.71(0.34-8.54)$ & 0.51 & $1.53(0.32-7.29)$ & 0.60 \\
\hline Macrolides & $57(53.8)$ & $6(50.0)$ & $1.16(0.32-4.20)$ & 0.82 & $1.30(0.34-4.98)$ & 0.70 \\
\hline Metronidazole & $41(38.7)$ & $6(50.0)$ & 0.63 (0.21-1.92) & 0.42 & $0.80(0.26-2.46)$ & 0.70 \\
\hline Fluoroquinolones & 35 (33.0) & $2(16.7)$ & $2.46(0.50-12.22)$ & 0.27 & 1.87 (0.40-8.82) & 0.43 \\
\hline
\end{tabular}


fecal droppings would strengthen this finding. Future research should consider if owning more chickens leads to an increase in human gut antibiotic resistance determinants. Despite limitations, this study supports other research findings suggesting that poultry are reservoirs for tetracycline resistance in humans. ${ }^{3}$

Although our findings suggest a relationship between poultry ownership and tetracycline resistance in children, more research is needed on this topic because the origins of AMR in the environment are not well understood. Strategies should be used to reduce human exposure to live poultry such as keeping chickens outside of living quarters, the sanitary disposal of chicken droppings, and improving antibiotic stewardship for domesticated animals. Investigating where crossover events are occurring between animals and humans is an important subject that could lead to crucial interventions to combat antibiotic resistance around the globe.

Received October 24, 2020. Accepted for publication February 8, 2021.

Published online March 22, 2021.

Financial support: This trial was supported by the National Eye Institute of the NIH under award K08EY026986 and by the Research to Prevent Blindness Career Development Award.

Disclosure: All authors submitted the ICMJE Form for Disclosure of Potential Conflicts of Interest.

Authors' addresses: Jessica M. Brogdon, Elodie Lebas, Lina Zhong, Cindi Chen, Thomas Lietman, Thuy Doan, and Catherine E. Oldenburg, Francis I Proctor Foundation, University of California, San Francisco, San Francisco, CA, E-mails: jessica.brogdon@ucsf.edu, elodie.Lebas@ucsf.edu, lina.zhong@ucsf.edu, cindi.chen@ucsf.edu, tom.lietman@ucsf.edu, thuy.doan@ucsf.edu, and catherine.oldenburg@ ucsf.edu. Ali Sié, Clarisse Dah, Lucienne Ouermi, and Boubacar Coulibaly, Centre de Recherche en Santé de Nouna, Nouna, Burkina Faso, E-mails: alisie@yahoo.fr, n.clarissedah@yahoo.fr, ouermil@yahoo.fr, and boubacar@fasonet.bf. Jeremy D. Keenan, F.I. Proctor Foundation and Department of Ophthalmology, University of California, San Francisco, San Francisco, CA, E-mail: jessica.brogdon@ucsf.edu.

\section{REFERENCES}

1. Johnson JR et al., 2007. Antimicrobial drug-resistant Escherichia coli from humans and poultry products, Minnesota and Wisconsin, 2002-2004. Emerg Infect Dis 13: 838-846.

2. Nikolich MP, Hong G, Shoemaker NB, Salyers AA, 1994. Evidence for natural horizontal transfer of tetQ between bacteria that normally colonize humans and bacteria that normally colonize livestock. Appl Environ Microbiol 60: 3255-3260.

3. Miles TD, McLaughlin W, Brown PD, 2006. Antimicrobial resistance of Escherichia coli isolates from broiler chickens and humans. BMC Vet Res 2: 7.

4. Rousham EK, Unicomb L, Islam MA, 2018. Human, animal and environmental contributors to antibiotic resistance in low- resource settings: integrating behavioural, epidemiological and one health approaches. Proc Biol Sci 285: 20180332.

5. WHO, 2014. Global Report on Surveillance: Antimicrobial Resistance. Geneva, Switzerland: World Health Organization.

6. Collignon P, Powers JH, Chiller TM, Aidara-Kane A, Aarestrup FM, 2009. World Health Organization ranking of antimicrobials according to their importance in human medicine: a critical step for developing risk management strategies for the use of antimicrobials in food production animals. Clin Infect Dis 49: 132-141.

7. Founou RC, Founou LL, Essack SY, 2017. Clinical and economic impact of antibiotic resistance in developing countries: a systematic review and meta-analysis. PLoS One 12: e0189621.

8. Roess AA et al., 2015. Household animal and human medicine use and animal husbandry practices in rural Bangladesh: risk factors for emerging zoonotic disease and antibiotic resistance. Zoonoses Public Health 62: 569-578.

9. Ngure F et al., 2019. Exposure to livestock feces and water quality, sanitation, and hygiene (WASH) conditions among caregivers and young children: formative research in rural Burkina Faso. Am J Trop Med Hyg 100: 998-1004.

10. Ercumen A, Prottas C, Harris A, Dioguardi A, Dowd G, Guiteras R, 2020. Poultry ownership associated with increased risk of child diarrhea: cross-sectional evidence from Uganda. Am J Trop Med Hyg 102: 526-533.

11. Oldenburg CE et al., 2018. Effect of commonly used pediatric antibiotics on gut microbial diversity in preschool children in Burkina Faso: a randomized clinical trial. Open Forum Infect Dis 5: ofy289.

12. Sie A et al., 2018. Effect of antibiotics on short-term growth among children in Burkina Faso: a randomized trial. Am J Trop Med Hyg 99: 789-796.

13. Sie $A$ et al., 2018. Dietary diversity and nutritional status among children in rural Burkina Faso. Int Health 10: 157-162.

14. Oldenburg CE et al., 2020. Gut resistome after oral antibiotics in preschool children in Burkina Faso: a randomized, controlled trial. Clin Infect Dis 70: 525-527.

15. Debela MD, Muyanja DM, Kakuhikire B, Baguma C, Bangsberg DR, Tsai AC, Weil AA, Lai PS, 2018. Acquisition and quantification of antimicrobial resistance genes in the gut microbiome of Ugandan women exposed to small-scale chicken farming. Open Forum Infect Dis 5: S366.

16. Molechan C, Amoako DG, Abia ALK, Somboro AM, Bester LA, Essack SY, 2019. Molecular epidemiology of antibioticresistant Enterococcus spp. from the farm-to-fork continuum in intensive poultry production in KwaZulu-Natal, South Africa. Sci Total Environ. 692: 868-878.

17. Kyakuwaire M, Olupot G, Amoding A, Nkedi-Kizza P, Basamba TA, 2019. How safe is chicken litter for land application as an organic fertilizer? A review. Int J Environ Res Public Health 16: E3521.

18. Mahmoud MAM, Abdel-Mohsein HS, 2019. Hysterical tetracycline in intensive poultry farms accountable for substantial gene resistance, health and ecological risk in Egypt- manure and fish. Environ Pollut 255: 113039.

19. Manyi-Loh C, Mamphweli S, Meyer E, Okoh A, 2018. Antibiotic use in agriculture and its consequential resistance in environmental sources: potential public health implications. Molecules 23: 795.

20. Ventola CL, 2015. The antibiotic resistance: part 1: causes and threats. P\&T 40: 277-283. 